\title{
Technical advances in endoscopic ultrasound-guided fiducial placement for the treatment of pancreatic cancer
}

Authors

Institutions
Disaya Chavalitdhamrong ${ }^{1}$, Christopher J. DiMaio ${ }^{2}$, Peter D. Siersema ${ }^{3}$, Mihir S. Wagh ${ }^{1}$

Division of Gastroenterology, University of Florida, Gainesville, Florida, United States

2 Division of Gastroenterology, Mount Sinai School of Medicine, New York, New York, United States

${ }^{3}$ Department of Gastroenterology, University Medical Center Utrecht, The Netherlands submitted:

20. December 2014 accepted after revision: 26. February 2015

\section{Bibliography}

DOI http://dx.doi.org/

10.1055/s-0034-1392274

Published online: 21.7.2015

Endosc Int Open 2015; 03:

E373-E377

(c) Georg Thieme Verlag KG

Stuttgart · New York

E-ISSN 2196-9736

\section{Corresponding author}

\section{Mihir S. Wagh, MD, FACG,}

\section{FASGE}

Interventional Endoscopy,

Division of Gastroenterology, University of Florida

1329 SW 16th Street, Suite

5251

Gainesville, Florida 32608

United States

Fax: +1-352-627-9002

mihir.wagh@medicine.ufl.edu
Radiation therapy has an important role in the treatment of locally advanced or metastatic pancreatic cancer and can be used alone or in conjunction with surgery and/or systemic chemotherapy. Because of the challenge of delivering an accurate and optimal radiation dose, image-guided radiation therapy can be used to improve targeting. Fiducial markers can be placed in the tumor and used for localization in patients undergoing image-guided radiation therapy. The safety

\begin{tabular}{ll}
\hline Abbreviations \\
$\nabla$ \\
EUS $\quad$ endoscopic ultrasound \\
IGRT image-guided radiotherapy \\
IMRT intensity-modulated radiotherapy
\end{tabular}

\section{Introduction}

$\nabla$

Radiation therapy has an important role in the treatment of locally advanced or metastatic pancreatic cancer and can be used alone or in conjunction with surgery and/or systemic chemotherapy. A major challenge for radiation therapy is respiratory organ motion. Fiducial markers can be used for tracking respiration to accurately measure the extent of target motion. These fiducials can be used for localization and are implanted at the tumor site as reference points to assist in targeting radiation beams. Fiducials can be placed surgically or percutaneously under ultrasound or computed tomography guidance or by endoscopic ultrasound (EUS). EUS, a minimally invasive approach, provides excellent visualization of the pancreas and therefore has been used for endoscopic placement of fiducials in pancreatic lesions. EUS-guided fiducial placement has been reported for mediastinal tumors, prostate cancer, and gastrointestinal malignancies, including pancreatic cancers, hepatic malignancies, cholangiocarcinomas, as well as esophageal, gastric and feasibility of endoscopic ultrasound (EUS)guided placement of fiducials has been assessed and reported for the management of pancreatic cancer. We herein review the technique, efficacy, and safety profile of EUS-guided fiducial placement. In addition, we highlight recent advances and technological upgrades in EUS-guided fiducial delivery systems for pancreatic cancer most relevant to practicing gastroenterologists and interventional endoscopists.

and colon cancers [ $1-6]$. However, this review focuses on EUS-guided fiducial placement for the treatment of pancreatic cancer, which is most relevant to practicing gastroenterologists and interventional endoscopists.

\section{Treatment of pancreatic cancer \\ $\nabla$}

Pancreatic adenocarcinoma is the second most common gastrointestinal malignancy and the sixth leading cause of cancer mortality in the United States [7]. The prognosis for patients with pancreatic cancer is poor with an overall fiveyear survival rate of $6 \%$. Although surgical resection can be curative, approximately $20 \%$ of patients are eligible for surgery at diagnosis because most patients present with locally advanced, inoperable disease where chemoradiation plays a role in neoadjuvant therapy, adjuvant treatment, or palliation. EUS provides important information about disease stage, including involvement of the vasculature and adjacent organ invasion, and regional lymph node metastasis. In addition, EUS is considered the procedure of choice for tissue diagnosis of pancreatic cancer. It can be used for therapeutic interventions such as fine-needle injection, biliary drainage, celiac plexus block or neurolysis, brachytherapy, fiducial placement, and emerging therapies with antitumor agents delivered directly into the tumor $[8,9]$. 

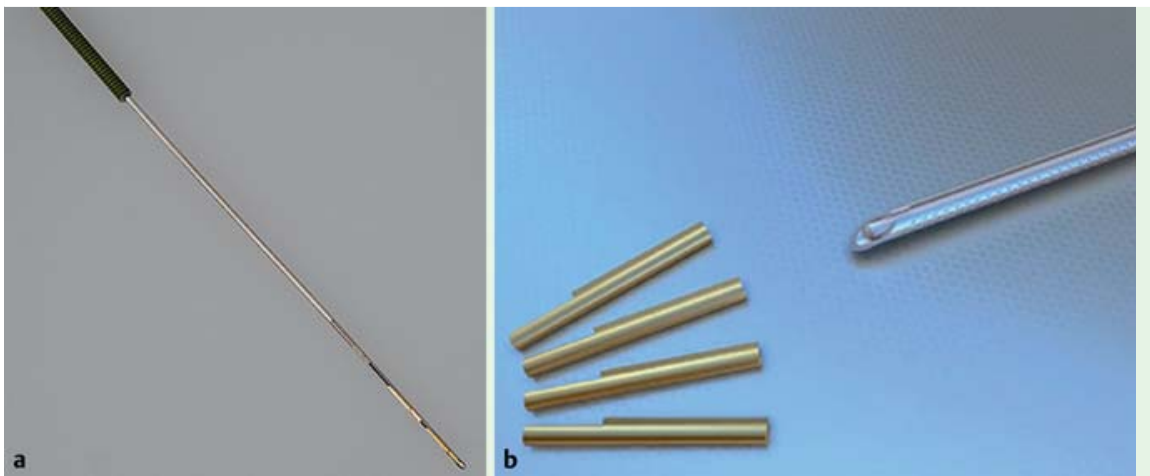

Fig. 1 Fiducial markers and a delivery needle.

Aggressive local treatment of pancreatic cancer leads to improved local control, which can increase overall survival [10]. For patients with locally advanced pancreatic cancer, chemoradiation enhances survival rates over chemotherapy alone [11, 12]. Several studies have demonstrated that aggressive local therapy with radiation dose escalation can improve local control. Radiation dose escalation has been attempted by way of intraoperative radiotherapy, stereotactic body radiotherapy, and intensity-modulated radiotherapy (IMRT) [13 - 15]. IMRT provides highprecision radiotherapy using a computer-controlled linear accelerator to deliver precise radiation doses to specific areas while maintaining or reducing healthy tissue exposure [16]. Imageguided radiotherapy (IGRT) is a novel technique that uses advanced imaging technology to verify target position before and during radiotherapy, and allows the delivery of precisely aimed radiation beams to tumors [17]. IGRT ensures that IMRT is delivered with optimal accuracy. IGRT relies on fiducial markers to target and track the location of the tumor in real time to ensure that radiation treatment is delivered to the tumor with pinpoint accuracy. The use of fiducial markers has become a viable option to facilitate accurate delivery of external beam radiation to a patient's treatment site during IMRT and IGRT treatments. This advancement in radiation therapy technique improves the chance of secondary resection, enhances local control for unresectable disease, and reduces symptoms related to local progression. In clinical practice, the decision for use of EUS-guided fiducial placement and IGRT is made jointly with oncologists, surgeons, and radiation oncologists.

\section{Fiducial markers \\ $\nabla$}

Fiducial markers are radiopaque spheres, coils, or seeds that are implanted into the target lesion in the pancreas for both localizing and tracking during radiotherapy (). These markers facilitate safe and accurate targeting of the tumor. Various types of fiducial markers and delivery systems ( $\bullet$ Fig. $\mathbf{1}$ a, b) have been developed [18]. A minimum of one to two fiducial markers are required to be placed either within the target lesion or within $1 \mathrm{~cm}$ of a small target lesion. Fiducial markers can be placed intraoperatively, percutaneously, or endoscopically $[19,20]$. Given the excellent visualization of lesions in proximity to the gastrointestinal tract, EUS offers an improved approach for fiducial placement ( $\bullet$ Fig.2). A higher ideal fiducial geometry for appropriate tracking has been reported with EUS when compared to surgery [21].

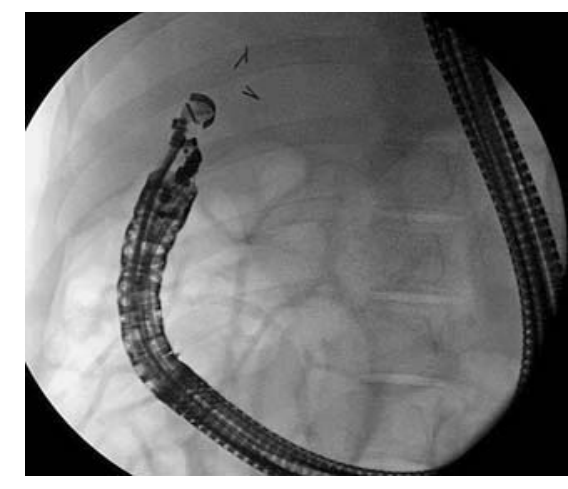

Fig. 2 Fluoroscopic view of the echoendoscope in a long position with four fiducials successfully deployed.

\section{Technique}

Fiducials can be delivered with 19 - or 22-gauge needles, or via multi-fiducial delivery systems $[2,3,5,21-31]$. The technique for fine-needle injection of fiducials is similar to that for fineneedle aspiration [22]. Traditional fiducials are cylindrical gold seeds, measuring 3 to $5 \mathrm{~mm}$ long and 0.75 to $1.2 \mathrm{~mm}$ in diameter. The new smaller and longer fiducial markers are $10 \mathrm{~mm}$ long and 0.28 or $0.35 \mathrm{~mm}$ in diameter. These new fiducials are available preloaded on a needle carrier delivery device for use with a 22gauge needle $[5,29]$. Comparative analysis of traditional fiducials vs coiled fiducials for pancreatic cancer using both 19-gauge and 22-gauge needles via EUS reported higher visibility for traditional fiducials without a difference in fiducial migration [23]. A 19gauge needle has been traditionally used, although a 22-gauge needle has also demonstrated feasibility, safety, and accuracy [5, 24]. A 22-gauge needle is potentially advantageous over a 19gauge needle because of its greater flexibility and smaller diameter, particularly when used in challenging anatomic locations or postsurgical anatomy.

\section{Fiducial placement in the needle.}

There are two basic techniques by which the fiducial marker can be loaded into the EUS needle. The most common technique employed is the back-loading method [25]. The stylet of the needle is withdrawn approximately $2-3 \mathrm{~cm}$. The fiducial delivery system, typically consisting of a fiducial marker loaded on a needle carrier, is then inserted "retrograde" into the needle tip ( $\bullet$ Fig.3). The fiducial is then deployed into the needle tip by removing the needle carrier. Finally, the tip of the EUS needle is plugged or sealed with a small amount of sterile bone wax to prevent inadvertent loss of the fiducial while advancing the needle down the accessory channel. A new variation of this method is the "wet-fill technique" where the EUS needle is submerged in sterile saline and the stylet is slowly retracted approximately $10 \mathrm{~cm}$. This effec- 


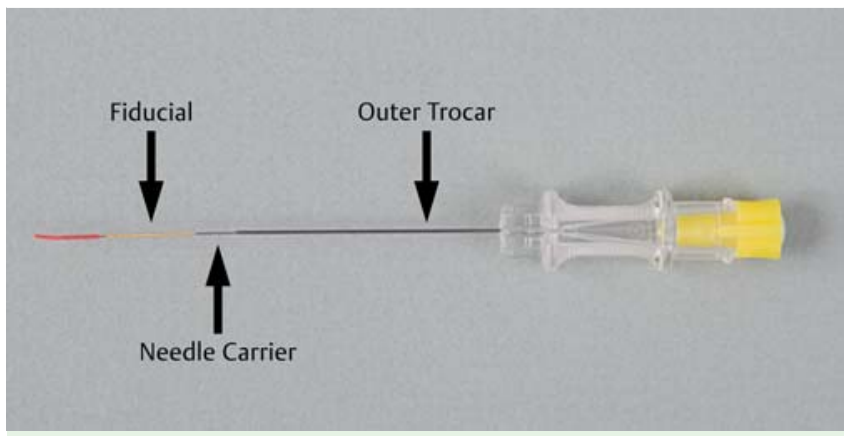

Fig. 3 Needle with fiducial marker.

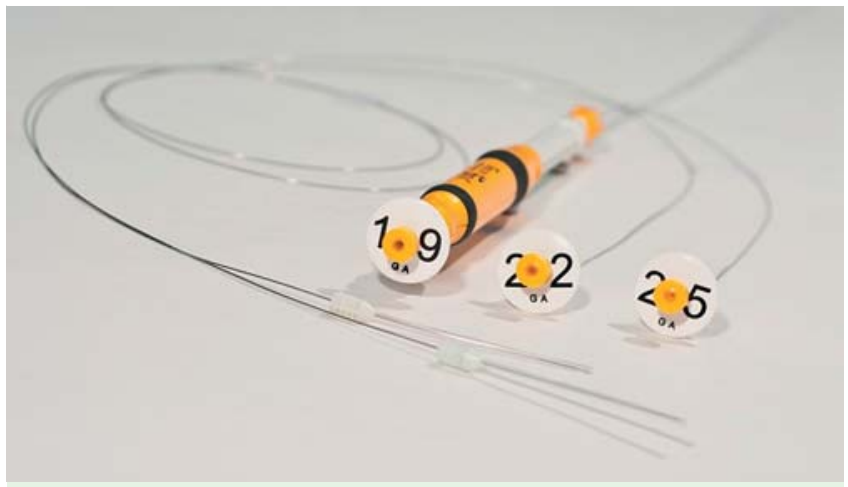

Fig. 4 The BNX needle aspiration system.

tively fills the distal end of the EUS needle with fluid [24], and the fiducial marker is then back-loaded into the EUS needle as previously described. Bone wax is not required to seal the needle tip because the fiducial is held in place within the needle via the surface tension of the saline. This technique may have potential advantages of avoiding failure of deployment related to bone wax and possible complication of granuloma formation following implantation of bone wax into tissue [26]. A potential disadvantage to using this back-loading method is the possibility of needle stick injuries. This is of particular concern when one EUS needle is reloaded between fiducial deployments. A solution to this problem is to have multiple preloaded needles readily available. Recently, the BNX needle aspiration system (Covidien, Dublin, Ireland) has been introduced for EUS fine-needle aspiration and EUS-guided fiducial placement ( $\bullet$ Fig. 4) [24]. It is engineered to improve clinical workflow by facilitating the passage of multiple needles through a single delivery system without removing the delivery system between needle passages. It allows rapid exchange of the needles while keeping the needle sheath and catheter in the echoendoscope. Therefore, having two or three preloaded needles can allow rapid deployment of multiple fiducials (this system allows placement of multiple fiducials through a single delivery system).

Another potential less risky approach to load the EUS needle is by using an antegrade or front-loading approach [27]. In this method, the stylet is removed entirely from the EUS needle. The needle tip is plugged with bone wax. A fiducial marker is then deployed into the top of the needle. The stylet is then reinserted and slowly advanced, thereby pushing the fiducial into the distal end of the needle tip. An advantage of this technique is that needle loading can be performed once the needle has been inserted into the target lesion. This precludes the need for plugging the tip with bone wax, and can allow for rapid deployment of multiple fiducials while the EUS needle is already in the echoendoscope.

\section{Fiducial deployment.}

Regardless of the method chosen to load the needle, all fiducials are typically deployed by advancing the stylet in the needle channel and pushing the stylet out of the needle tip.

\section{Efficacy}

EUS evaluation of pancreatic cancer has been reported as an independent predictor of improved survival in patients with locally advanced pancreatic cancer [28]. EUS-guided placement of fiducial markers before targeted radiation therapy is a novel clinical application for cancer management $[8,9]$. EUS-guided fiducial placement provides the advantage of being performed during the same session as EUS examination for staging. In 2006, Pishvaian et al. published the first report in which fiducials were successfully placed via EUS guidance in six of seven pancreatic cancer patients [26]. Sanders and colleagues reported a success rate of $90 \%$ for EUS-guided fiducial placement in 51 patients [29]. These authors used a 19-gauge needle with a pushing stylet technique for fiducial insertion. They noted difficulty in fiducial placement in patients who had undergone a prior pancreaticoduodenectomy. Park et al. reported an $88 \%$ success rate of EUSguided gold fiducial insertion in 57 patients [30]. These authors back-loaded the fiducials into a 19-gauge needle and fixed the fiducials to the tip of the needle with bone wax. A sterile water injection technique was used to insert the fiducials instead of pushing with a stylet. This technique was employed to prevent coiling of the fiducials. In summary, the feasibility of EUS-guided fiducial placement with or without fluoroscopy for locally advanced and recurrent pancreatic cancer has been reported with technical

Table 1 Efficacy and safety of endoscopic ultrasound-guided fiducial placement.

\begin{tabular}{|c|c|c|c|c|}
\hline Study & Patients (\#) & Cancer (type) & Efficacy (\%) & Adverse events (\# of patients) \\
\hline Pishvaian AC et al. [3] & 13 & Mediastinal and abdominal malignancies & 84.6 & Infectious complication (1) \\
\hline Varadarajulu S et al. [31] & 9 & Pancreatic cancer & 100 & None \\
\hline Ammar T et al. [27] & 13 & Abdominal malignancies & 100 & None \\
\hline Park WG et al. [30] & 57 & Pancreatic cancer & 94 & $\begin{array}{l}\text { Needle malfunction ( } 1 \text { ), and minor } \\
\text { bleeding ( } 1 \text { ) }\end{array}$ \\
\hline Sanders MK et al. [29] & 51 & Pancreatic cancer & 90 & Mild pancreatitis ( 1 ) \\
\hline DiMaio C] et al. [5] & 30 & Gastrointestinal malignancies & 97 & Infectious complication ( 1 ) \\
\hline Choi JH et al. [2] & 32 & Pancreatic and hepatic malignancy & 100 & Mild pancreatitis (1) \\
\hline Majumder S et al. [21] & 77 & Pancreatic cancer & 90 & $\begin{array}{l}\text { Abdominal pain (3), vomiting ( } 1 \text { ), } \\
\text { mild pancreatitis ( } 1 \text { ) }\end{array}$ \\
\hline Davila Fajardo R et al. [32] & 23 & Pancreatic cancer & 100 & Minor bleeding (1) \\
\hline
\end{tabular}




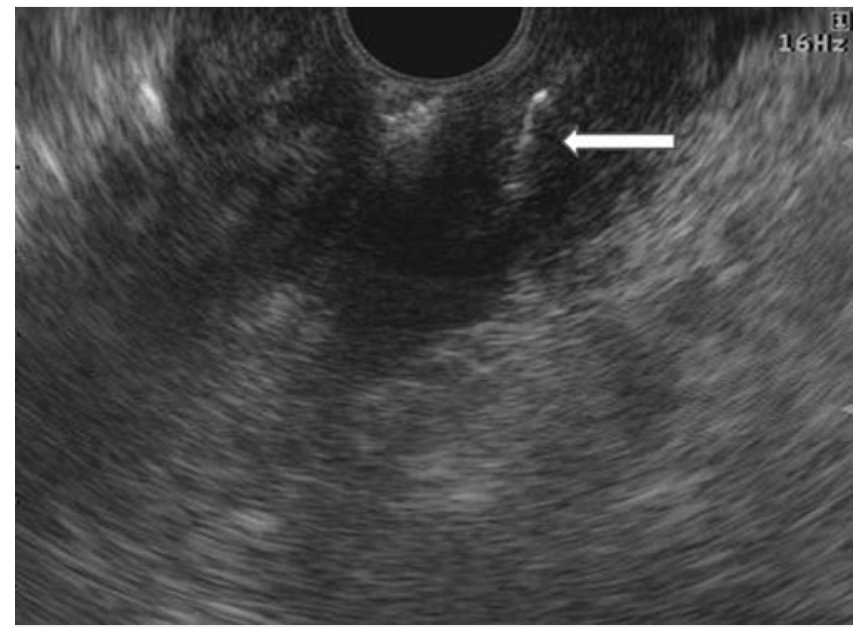

Fig.5 Endosonographic image of needle with fiducial.

success rates of $85 \%$ to $100 \%[2,3,5,21,27,29-32]$. The reported data on efficacy and safety of EUS-guided fiducial placement are shown in the Table 1.

\section{Technical difficulties}

The use of multiple fiducials can reduce the impact of organ deformation and fiducial migration during radiotherapy. Multiple needle insertions of a single fiducial delivery system are required to deliver multiple fiducials. Delivery of multiple fiducials requires removal of the needle and manual reloading of each fiducial. It is therefore desirable to provide multiple fiducials in a single needle that can be introduced in a single pass and in a controlled serial manner. This would help overcome the difficulties in advancing fiducials with the echoendoscope in the duodenum where sharp angulation and torque are needed to target pancreatic head and uncinate lesions.

As therapeutic applications of EUS continue to expand, improved accessories are required to meet treatment needs. It is important to consider the development of flexible multi-fiducial delivery needles that can deploy multiple fiducials in one pass. A new multi-fiducial delivery system that has been recently introduced is a 22-gauge needle with a holding chamber at the needle tip preloaded with four gold fiducials and adjustable locking handle. Each gold marker is $5 \mathrm{~mm}$ long. The flexible sheath of the delivery needle has a 5.2-French outer diameter compatible with the accessory channel of an echoendoscope [33]. We recently reported that this delivery system provided quick, easy, and accurate fiducial deployment without adverse events in a live porcine model [33]. Our human study is currently enrolling patients with pancreatic cancer for further evaluation of this new delivery system.

\section{Adverse events}

Reported complications include acute pancreatitis, minor bleeding, fever, vomiting, abdominal pain, and elevated liver enzymes $[2,3,5,21,29,30,32]$ as shown in the Table 1 . Some authors have suggested use of prophylactic antibiotics [27] during EUSguided fiducial placement and further studies are needed to validate this recommendation. The rate of fiducial migration is relatively low (7\%) and no migration-related complications have been reported [29].

\section{Future directions}

The primary drawback of EUS-guided fiducial placement is the lack of dedicated accessories designed specifically for fiducial placement. Multi-fiducial delivery systems are therefore a welcome upgrade in this field. More flexible fiducial delivery systems that are unlikely to bend or kink during deployment with the echoendoscope in the long position, or in an angulated and torqued configuration, are desirable to allow fiducial placement in the pancreatic head and uncinate. Enhanced visualization of the needle and fiducial during EUS is necessary to allow fiducial placement solely under endosonographic guidance without the use of fluoroscopy, thus minimizing radiation exposure ( $\bullet$ Fig.5). Increasing the awareness of this application of EUS among oncologists, radiation oncologists, surgeons, gastroenterologists, and interventional endoscopists is vital as this technology can become an essential part of the multidisciplinary approach to pancreatic cancer treatment.

\section{Conclusion}

\section{$\nabla$}

Application of image-guided radiation therapy to locally advanced unresectable pancreatic cancer requires the use of fiducials to track the precise location of the tumor. Fiducial placement under EUS guidance has been reported as safe, feasible, and effective. Recent advances in endoscopic accessories for EUS-guided fiducial placement are promising and will allow more endoscopists to adopt this modality in their therapeutic EUS armamentarium.

\section{Competing interests: None}

\section{References}

1 Fernandez DC, Hoffe SE, Barthel JS et al. Stability of endoscopic ultrasound-guided fiducial marker placement for esophageal cancer target delineation and image-guided radiation therapy. Pract Radiat Oncol 2013; 3 : $32-39$

2 Choi JH, Seo DW, Park do $H$ et al. Fiducial placement for stereotactic body radiation therapy under only endoscopic ultrasonography guidance in pancreatic and hepatic malignancy: practical feasibility and safety. Gut Liver 2014; 8: 88-93

3 Pishvaian AC, Collins B, Gagnon G et al. EUS-guided fiducial placement for CyberKnife radiotherapy of mediastinal and abdominal malignancies. Gastrointest Endosc 2006; 64: $412-417$

4 Suzuki R, Irisawa A, Bhutani MS. Endoscopic ultrasound-guided oncologic therapy for pancreatic cancer. Diagn Ther Endosc 2013; 2013: 157581

5 DiMaio CJ, Nagula S, Goodman KA et al. EUS-guided fiducial placement for image-guided radiation therapy in GI malignancies by using a 22gauge needle (with videos). Gastrointest Endosc 2010; 71: 1204-1210

6 Yang J, Abdel-Wahab M, Ribeiro A. EUS-guided fiducial placement after radical prostatectomy before targeted radiation therapy for prostate cancer recurrence. Gastrointest Endosc 2011; 73: 1302 - 1305

7 Siegel R, Ma J, Zou Z et al. Cancer statistics, 2014. CA Cancer J Clin 2014; 64: 9-29

8 Othman MO, Wallace MB. The role of endoscopic ultrasonography in the diagnosis and management of pancreatic cancer. Gastroenterol Clin North Am 2012; 41: 179-188

9 Jin Z, Chang KJ. Endoscopic ultrasound-guided fiducial markers and brachytherapy. Gastrointest Endosc Clin N Am 2012; 22: 325 - 331, x

10 Gutt R, Liauw SL, Weichselbaum RR. The role of radiotherapy in locally advanced pancreatic carcinoma. Nat Rev Gastroenterol Hepatol 2010; 7: $437-447$

11 Willett CG, Czito BG, Bendell JC et al. Locally advanced pancreatic cancer. J Clin Oncol 2005; 23: 4538-4544

12 Sultana A, Tudur Smith C, Cunningham D et al. Systematic review, including meta-analyses, on the management of locally advanced pan- 
creatic cancer using radiation/combined modality therapy. Br J Cancer 2007; 96: $1183-1190$

13 Willett CG, Del Castillo CF, Shih HA et al. Long-term results of intraoperative electron beam irradiation (IOERT) for patients with unresectable pancreatic cancer. Ann Surg 2005; 241: 295 - 299

14 Chang DT, Schellenberg D, Shen J et al. Stereotactic radiotherapy for unresectable adenocarcinoma of the pancreas. Cancer 2009; 115: 665 672

15 Milano MT, Chmura SJ, Garofalo MC et al. Intensity-modulated radiotherapy in treatment of pancreatic and bile duct malignancies: toxicity and clinical outcome. Int J Radiat Oncol Biol Phys 2004; 59: 445 - 453

16 Yovino S, Poppe $M$, Jabbour $S$ et al. Intensity-modulated radiation therapy significantly improves acute gastrointestinal toxicity in pancreatic and ampullary cancers. Int J Radiat Oncol Biol Phys 2011; 79: 158 - 162

17 Fuss $M$, Wong $A$, Fuller $C D$ et al. Image-guided intensity-modulated radiotherapy for pancreatic carcinoma. Gastrointest Cancer Res 2007; 1: $2-11$

18 Habermehl D, Henkner K, Ecker $S$ et al. Evaluation of different fiducial markers for image-guided radiotherapy and particle therapy. J Radiat Res 2013; 54: i61 - i68

19 Kothary N, Heit JJ, Louie JD et al. Safety and efficacy of percutaneous fiducial marker implantation for image-guided radiation therapy. J Vasc Interv Radiol 2009; 20: 235-239

$20 \mathrm{Kim} \mathrm{JH}$, Hong SS, Kim JH et al. Safety and efficacy of ultrasound-guided fiducial marker implantation for CyberKnife radiation therapy. Korean J Radiol 2012; 13: 307-313

21 Majumder S, Berzin TM, Mahadevan A et al. Endoscopic ultrasoundguided pancreatic fiducial placement: how important is ideal fiducial geometry? Pancreas 2013; 42: 692-695

22 Fuccio L, Attili F, Vanella $G$ et al. Interventional endoscopic ultrasonography. Curr Treat Options Gastroenterol 2014; 12: 183-210

23 Khashab MA, Kim KJ, Tryggestad EJ et al. Comparative analysis of traditional and coiled fiducials implanted during EUS for pancreatic cancer patients receiving stereotactic body radiation therapy. Gastrointest Endosc 2012; 76: $962-971$
24 Khara HS, Pineda-Bonilla JJ, Chaput KJ et al. Endoscopic ultrasoundguided placement of fiducial markers using a novel "wet-fill technique" without a bone wax seal. Endoscopy 2013; 45: E426 - 427

25 Owens DJ, Savides TJ. EUS placement of metal fiducials by using a backloaded technique with bone wax seal. Gastrointest Endosc 2009; 69: 972-973

26 Anfinsen OG, Sudmann B, Rait M et al. Complications secondary to the use of standard bone wax in seven patients. J Foot Ankle Surg 1993; 32: $505-508$

27 Ammar T, Cote GA, Creach KM et al. Fiducial placement for stereotactic radiation by using EUS: feasibility when using a marker compatible with a standard 22-gauge needle. Gastrointest Endosc 2010; 71: $630-633$

28 Ngamruengphong S, Li F, Zhou Y et al. EUS and survival in patients with pancreatic cancer: a population-based study. Gastrointest Endosc 2010; $72: 78-83$

29 Sanders $M K$, Moser AJ, Khalid A et al. EUS-guided fiducial placement for stereotactic body radiotherapy in locally advanced and recurrent pancreatic cancer. Gastrointest Endosc 2010; 71: 1178 - 1184

30 Park WG, Yan BM, Schellenberg $D$ et al. EUS-guided gold fiducial insertion for image-guided radiation therapy of pancreatic cancer: 50 successful cases without fluoroscopy. Gastrointest Endosc 2010; 71: 513 518

31 Varadarajulu S, Trevino JM, Shen $S$ et al. The use of endoscopic ultrasound-guided gold markers in image-guided radiation therapy of pancreatic cancers: a case series. Endoscopy 2010; 42: 423-425

32 Davila Fajardo R, Lekkerkerker SJ, van der Horst A et al. EUS-guided fiducial markers placement with a 22-gauge needle for image-guided radiation therapy in pancreatic cancer. Gastrointest Endosc 2014; 79: $851-855$

33 Draganov PV, Chavalitdhamrong D, Wagh MS. Evaluation of a new endoscopic ultrasound-guided multi-fiducial delivery system: a prospective non-survival study in a live porcine model. Dig Endosc 2013; 25: $615-621$ 